\title{
Profile of Enfortumab Vedotin in the Treatment of Urothelial Carcinoma: The Evidence to Date
}

This article was published in the following Dove Press journal:

Drug Design, Development and Therapy

\author{
Mohamad Moussa' \\ Athanasios Papatsoris ${ }^{2}$ \\ Mohamed Abou Chakra' \\ Athanasios Dellis ${ }^{2,3}$ \\ 'Department of Urology, Al Zahraa \\ Hospital, University Medical Center, \\ Lebanese University, Beirut, Lebanon; \\ ${ }^{2} 2$ nd Department of Urology, School of \\ Medicine, Sismanoglio Hospital, National \\ and Kapodistrian University of Athens, \\ Athens, Greece; ${ }^{3}$ Department of Surgery, \\ School of Medicine, Aretaieion Hospital, \\ National and Kapodistrian University of \\ Athens, Athens, Greece
}

\begin{abstract}
Nowadays the therapeutic landscape for advanced and metastatic urothelial carcinoma continues to evolve. The recent regulatory approval of enfortumab vedotin (EV) for the treatment of advanced urothelial cancer confirms the evolving role of antibody-drug conjugates. EV demonstrates a favorable profile in heavily pretreated patients with locally advanced or metastatic urothelial carcinoma. Early survival reports demonstrate a significant antitumor effectiveness along with a rather acceptable safety profile in a difficult-to-treat population.
\end{abstract}

Keywords: enfortumab vedotin, bladder cancer, metastasis, urothelial cancer

\section{Introduction}

Bladder cancer (BC) is the 8th most commonly diagnosed cancer in males, dropping to 12th when both genders are considered, ${ }^{1}$ with approximately 550,000 new cases and 200,000 BC-related deaths in 2018. ${ }^{2}$ Southern Europe presents the highest age-standardized rates (ASRs) in BC incidence in both genders, with 26.5 per 100,000 for males and 5.5 per 100,000 for females, respectively. About $25 \%$ of cases present with muscle-invasive $\mathrm{BC}$ (MIBC) and approximately half of them relapse after radical cystectomy (RC), depending on the pathological stage of the primary tumour and the nodal status. ${ }^{3}$ Approximately $5 \%$ of patients have metastatic disease at the time of diagnosis. ${ }^{4}$ Before the development of effective chemotherapy, patients with metastatic $\mathrm{BC}(\mathrm{mBC})$ had a median survival rarely exceeding three to six months. ${ }^{5}$ Cisplatin-based chemotherapy has been the standard of care for more than 30 years as first-line treatment in cisplatin-eligible patients. The combination of methotrexate, vinblastine, adriamycin plus cisplatin (MVAC) was equivalent to gemcitabine plus cisplatin (GC) in terms of overall survival (OS) prolongation. ${ }^{6}$

The high-dose intensity MVAC in combination with G-CSF is proven better in terms of safety and efficacy compared with standard MVAC, although median survival is similar. ${ }^{7}$ Long-term survival seems to be significantly dependent on the metastatic sites: in LN-only disease, 5-year survival reaches $20.9 \%$ compared to only $6.8 \%$ for patients with visceral metastases. ${ }^{6}$

In patients unfit for cisplatin, checkpoint inhibitors (CPIs) suggest promising alternatives along with carboplatin combination chemotherapy. In case of disease progress, the only second-line treatment option with relatively good results is CPI pembrolizumab. ${ }^{3}$ Several novel agents are tested in Phase 1 and 2 trials for second-line therapy in order to further prolong OS and improve severe toxicity issues that
Correspondence: Athanasios Papatsoris 2nd Department of Urology, School of Medicine, Sismanoglio Hospital, National and Kapodistrian University of Athens, Athens, Greece

Fax +302/32058605

Email agpapatsoris@yahoo.gr 
characterize established treatment for metastatic disease. Enfortumab vedotin (EV) is a promising drug recently approved by the FDA for the treatment of adult patients with locally advanced or $\mathrm{mBC}$ who have previously received a programmed death receptor-1 (PD-1) or programmed death ligand 1 (PD-L1) inhibitor and a platinum-containing chemotherapy. ${ }^{8}$ Herein, we present recent evidence on the profile, effectiveness and safety of EV.

\section{Enfortumab Vedotin}

\section{Enfortumab Vedotin Outline and}

\section{Mechanism of Action}

Enfortumab vedotin (EV) is a novel, intravenously (iv) administered, fully humanized, monoclonal antibodydrug conjugate (ADC) designed for the treatment of neoplasms with cells that express Nectin-4, also known as poliovirus receptor-related protein 4 (PVLR4). ${ }^{9}$ Nectin-4 is an adhesion immunoglobulin-like transmembrane molecule, consisted of extra- and intracellular domains as well as a transmembrane helix ${ }^{10}$ that plays a role in several stages of cellular cycle. Nectin-4 is highly expressed in urothelial cancer (UC) cells, especially in BC cells but poorly in normal cells and is associated with poor prognosis. ${ }^{11,12}$ Of note there are two versions of EV, the hybridoma (AGS-22M6E) and the Chinese Hamster Ovary (CHO or AGS-22CE) version, both of which were proven to induce cell death in vitro. ${ }^{12}$

Enfortumab suggests the anti-Nectin-4 monoclonal antibody part attached via a cathepsin-cleavable linker to vedotin. Vedotin refers to the monomethyl auristatin E (MMAE), a microtubule-disrupting agent, that is delivered and selectively bound to Nectin- 4 expressing BC cells. ${ }^{13}$ The resulting ADC-Nectin-4 complex is internalized into the BC cells and then the valine-citrulline (Val-Cit) dipeptide linker is recognized and cleaved by cathepsin-B in the cells of the neoplasm, where the conjugated cytotoxic MMAE is selectively released. As a result, the microtubule networks are disrupted leading to apoptotic death. ${ }^{12}$

$\mathrm{EV}$ was granted accelerated approval by the FDA on 18 December 2019. ${ }^{14}$ According to NCCN guidelines on $\mathrm{mBC}, \mathrm{EV}$ is recommended as a preferred second or subsequent-line systemic therapy option in previously treated patients in the setting of a clinical trial, ${ }^{15}$ while the EAU Guidelines consider EV as a promising drug for second or later-line therapy. ${ }^{3}$

\section{Clinical Trials \\ EV-I0I (ASG-22CE-I3-2) (NCT0209I999)}

EV was initially assessed for safety/tolerability and antitumor activity in EV-101 trial (ASG-22CE-13-2) (NCT02091999), a phase 1, open-label, North American multi-center, dose escalation/dose expansion study in patients with Nectin-4-positive tumors (including metastatic UC (mUC) and especially $\mathrm{mBC}$ ) who were heavily pretreated with at least 1 prior chemotherapy regimen and/ or PD-L1 inhibitor, including a cohort of patients with mUC who received prior anti-PD-L1 therapy. ${ }^{16}$ Twentyfive patients were enrolled in the dose escalation phase, most of them with primary tumors in the bladder, renal pelvis, or ureter $(n=21) ; 110$ patients $(71 \%$ of the 155 totally enrolled patients) had $\mathrm{mBC}$ and were treated with EV $1.25 \mathrm{mg} / \mathrm{kg}$ iv. Patients received escalating doses of EV up to $1.25 \mathrm{mg} / \mathrm{kg}$ on days 1,8 , and 15 of every 28 -day cycle. Primary objectives of the study were the evaluation of safety/tolerability and pharmacokinetics; antitumor activity was a secondary objective. Enrolled patients with mUC $(n=155)$ were heavily pretreated, with $96 \%$ of them with prior platinum-based chemotherapy and $29 \%$ receiving at least 3 lines of prior treatment. Maximum tolerated dose (MTD) of EV was not established; however, the recommended Phase 2 dose was $1.25 \mathrm{mg} / \mathrm{kg}$. The most common treatment-related adverse events (TRAEs) were rash, peripheral neuropathy, fatigue, alopecia, and nausea, with most of them grade 1-2 in severity. Among the 112 patients with mUC treated with single-agent EV $1.25 \mathrm{mg} /$ $\mathrm{kg}$, the investigator-assessed confirmed objective response rate (ORR) was 43\%, while the duration of response (DOR) was 7.4 months. Median OS reported was 12.3months. Similar ORR and estimated median OS were observed in patients older than 75 years of age with and without prior anti-PD-L1 treatment, liver metastases, or upper-tract urothelial cancer (UTUC). It was therefore concluded that EV was generally well tolerated and provided clinically meaningful and durable responses in patients with mUC with encouraging survival data.

\section{EV-20I (NCT03219333)}

Because of the strength of the aforementioned data, singleagent $\mathrm{EV}$ has been investigated in patients with locally advanced/mUC previously treated with platinum-based chemotherapy and CPI therapy in a pivotal phase 2 study (EV-201) (NCT03219333). ${ }^{17}$ EV-201 is a global twocohort, single-arm, phase 2 study, designed to establish the efficacy and safety of EV in 125 eligible patients 
from USA and Japan with locally advanced or mUC. In total, $90 \%$ of enrolled patients suffered from metastatic visceral disease, while liver metastases were present in $40 \%$ of patients. Cohort 1 enrolled patients who were previously treated with both platinum chemotherapy and an anti-PD-1/L1 therapy, whereas Cohort 2 continues to enroll patients who were previously treated only with CPI therapy. Similar to EV-101, the median number of previous therapies was 3 (range, $1-6$ ). EV $1.25 \mathrm{mg} / \mathrm{kg}$ was administered iv on days 1,8 , and 15 of every 28 -day cycle. The primary end point was ORR per Response Evaluation Criteria in Solid Tumors (RECIST) version 1.1 assessed by blinded independent central review. Key secondary end points were DOR, progression-free survival (PFS), OS, safety, and tolerability. The median follow-up was 10.2 months (range: 0.5 to 16.5 months). Confirmed ORR by blinded independent reviewers was $44 \%$ (range: $35.1 \%$ to $53.2 \%$ ), including $12 \%$ complete remissions. Similar responses were observed in prespecified subgroups, such as in poor prognosis patients with liver metastases or nonresponders to previously received anti-PD-1/L1 therapy. Median DOR was 7.6 months (range: 0.95 to 11.3 months). The most common TRAEs were fatigue (50\%), any peripheral neuropathy (50\%), alopecia (49\%), any rash (48\%), decreased appetite (44\%), and dysgeusia (40\%), with approximately $90 \%$ of TRAEs grade $1-2$. It was concluded that EV demonstrated a clinically meaningful response rate with a manageable and tolerable safety profile in patients with locally advanced or mUC who were previously treated with platinum and anti-PD-1/ L1 therapies.

\section{EV-30I (NCT03474I07)}

It is an open-label, multi-center, randomized, Phase 3 study to compare the OS of 608 participants with locally advanced or mUC treated with EV to the OS of participants treated with investigator's choice of chemotherapy, who showed progression of disease on either platinumbased chemotherapy or immunotherapy. Participants receive iv EV on days 1,8 and 15 of each 28-day cycle or either iv docetaxel, vinflunine or paclitaxel on day 1 of every 21-day cycle. OS is the primary outcome of the study, while the secondary outcomes are PFS, ORR, the DCR and DOR per RECIST V1.1 and the assessment of safety and tolerability, the quality of life (QOL) and patient-reported outcomes (PRO) parameters.

Recently, a press release reported that EV-301 met its primary endpoint of OS compared to chemotherapy and was considered to be positive. The study results were carefully reviewed by an independent Data Monitoring Committee following a planned interim analysis. EV was proven to significantly improve OS and PFS ( $\mathrm{p}=0.001$ and $\mathrm{p}<0.00001$, respectively). Reported TRAEs were rash, hyperglycemia, decreased neutrophil count, fatigue, anemia and decreased appetite (the most frequent Grade 3 or greater AEs occurring in more than $5 \%$ of patients). ${ }^{18}$

\section{EV-I03 (NCT03288545)}

It is a Phase1/2 randomized, multi-cohort, open-label, multi-center dose-escalation/dose-expansion study of iv EV as monotherapy or in combination with anticancer therapies such as chemotherapy or CPI for the treatment of locally advanced or mUC with estimated enrollment of 407 patients. In the dose-escalation phase, the combination of EV plus pembrolizumab as first-line or second-line in cisplatin-ineligible patients was assessed, while in the dose-expansion phase there were several cohorts examining EV combined with pembrolizumab, cisplatin, carboplatin or gemcitabine. The primary goal of the study is to determine the safety, tolerability, and efficacy of EV alone or in the aforementioned combinations. Initial results in 45 cisplatin-ineligible patients with $\mathrm{mUC}$ received $1.25 \mathrm{mg} / \mathrm{kg}$ EV on days 1 and 8 plus $200 \mathrm{mg}$ pembrolizumab on day 1 every 3 weeks, showed a preliminary confirmed ORR per RECIST 1.1 of $62 \%$ (14\% with complete and $48 \%$ with partial response, respectively), while $90 \%$ was the disease control rate (DCR). The most common TRAEs were fatigue $(66 \%$, with $14 \%$ of the cases at least Grade 3$)$, decreased appetite $(52 \%, 0 \% \geq$ Grade 3$)$, peripheral neuropathy of any type in $(52 \%, 3 \% \geq$ Grade 3$)$, alopecia $(45 \%)$, rash of any type $(45 \%, 14 \% \geq$ Grade 3$)$, diarrhea $(41 \%, 3 \%$ $\geq$ Grade 3 ) and immune-related events that needed steroids (17\%, 10\% $\geq$ Grade 3 ). A total of $7 \%$ of patients discontinued treatment with $\mathrm{EV}+$ pembrolizumab due to AEs (lipase increase, multi-organ failure). ${ }^{19}$

\section{NCT03606I74}

It is a phase 2, non-randomized, multi-cohort, open-label, multicenter study with estimated enrollment of 425 participants previously treated with CPI and platinum-based chemotherapy. This study examines the safety and anticancer activity of $\mathrm{EV}$ given iv as monotherapy and in combination with other anticancer therapies as first line (1L) and second line (2L) treatment for patients with UC. The primary objective is ORR. There are 2 portions in the study - a lead-in dose escalation portion and a dose 
expansion portion. In the dose escalation portion, patients are treated with up to 4 dose levels of sitravatinib (orally once per day continuously in 21-day cycles at $35 \mathrm{mg}$, $50 \mathrm{mg}, 70 \mathrm{mg}$ or $100 \mathrm{mg}$ ) in combination with pembrolizumab (200 mg over $30 \mathrm{~min}$ iv infusion every 3 weeks) and up to 2 dose levels of EV ( $30 \mathrm{~min}$ iv infusion on day 1 and day 8 in 21 -day cycles at $1 \mathrm{mg} / \mathrm{kg}$ or $1.25 \mathrm{mg} / \mathrm{kg}$ ) combination in order to determine the recommended doses to be used. These doses will be further studied in the dose expansion portion. The primary goal of the study is to determine the safety, tolerability, and efficacy of EV alone and in combination with pembrolizumab and/or chemotherapy. The early efficacy results of the study have demonstrated encouraging activity with a safety profile that appears manageable and tolerable. The addition of sitravatinib to the EV-pembrolizumab combination might further augment clinical activity by selectively inhibiting key molecular and cellular pathways strongly implicated in CPI resistance. The trial is still recruiting patients and has no results yet.

\section{EV-202 (NCT04225 I I7)}

It is an open-label, multicenter, multicohort, nonrandomized phase 2 study to evaluate EV in subjects with previously treated locally advanced or metastatic malignant solid tumors, with estimated enrollment of 240 participants. The primary purpose of this study is to determine the antitumor activity of EV as measured by confirmed ORR.

The study will also assess other measures of antitumor activity such as OS, safety and tolerability of EV and will consist of 3 periods: screening/baseline, treatment and follow-up. Screening/baseline period will take place up to 28 days prior to the first dose of study treatment. In the treatment period, starting at cycle 1, participants will receive $\mathrm{EV}$ on days 1,8 , and 15 every 28 -day cycle until one of the treatment discontinuation criteria are met. Disease assessment will be performed at screening/baseline and repeated every 8 weeks (56 days \pm 7 days) from the first dose of study treatment throughout the study until the participant has radiologically confirmed disease progression, initiates a new subsequent anticancer therapy, dies, withdraws consent, is lost to follow-up or the study closes, whichever occurs first.

Participants who discontinue study treatment for reasons other than radiologically confirmed disease progression by RECIST Version 1.1 will enter into a post-treatment followup period and continue to receive imaging scans every 8 weeks (56 days \pm 7 days) until the subject has radiologically confirmed disease progression, initiates a new anticancer therapy, dies, withdraws consent, is lost to follow-up or the study closes, whichever occurs first.

After 1 year on study treatment, the frequency of disease assessment will be reduced to every 12 weeks ( 84 days \pm 7 days).

After radiologically confirmed disease progression or initiation of subsequent anticancer therapy, whichever occurs first, participants will be contacted every 12 weeks in the long-term follow-up period for survival status until death, withdrawal of consent, lost to follow-up or study closure, whichever occurs first.

\section{EV-302 (NCT04223856)}

It is an open-label, randomized, controlled phase 3 study of EV plus pembrolizumab with (Arm C) or without chemotherapy (Arm A) versus standard of care gemcitabine plus platinum-containing chemotherapy (Arm B) in 760 patients with previously untreated locally advanced or mUC.

EV may be administered for an unlimited number of cycles and pembrolizumab for a maximum of 35 cycles or until a protocol defined reason for study discontinuation occurs, whichever is first. Cisplatin or carboplatin plus gemcitabine may be administered for a maximum of 6 cycles or a protocol-defined reason for study discontinuation occurs, whichever is first.

The primary outcomes are PFS per RECIST v1.1 and OS and secondary outcomes are ORR, DOR, DCR and TRAEs.

Recently, based on promising data on ORR from chemotherapy + PD-1/PD-L1 combinations with a manageable safety profile, the study was modified to remove Arm C. The updated 2-arm design randomizes patients to EV plus pembrolizumab or gemcitabine plus cisplatin/carboplatin in 1:1 ratio with the following stratification factors: cisplatin eligibility, PD-L1 expression and liver metastases. ${ }^{20}$

\section{MK-3475-905/KEYNOTE-905/EV-303 (NCT03924895)}

This is a randomized phase 3 study evaluating $\mathrm{RC}$ with either perioperative pembrolizumab or EV plus pembrolizumab versus $\mathrm{RC}$ alone in cisplatin-ineligible participants with MIBC with estimated enrollment of 836 patients. Participants receive 3 preoperative cycles of EV $(1.25 \mathrm{mg} / \mathrm{kg}$ iv, given on days 1 and 8 of each 21 -day 
Table I Clinical and Ongoing Trials on EV

\begin{tabular}{|c|c|c|c|c|c|}
\hline Trial [Ref] & Phase & Patients & Inclusion Criteria & Endpoints & Outcomes \\
\hline $\begin{array}{l}\text { EV-IOI } \\
\text { NCT0209199916 }\end{array}$ & 1 & 155 & $\begin{array}{l}\text { Patients with at least I prior } \\
\text { chemotherapy regimen and/ } \\
\text { or PD-LI inhibitor. }\end{array}$ & $\begin{array}{l}\text { Primary objectives: evaluation of } \\
\text { safety/tolerability and } \\
\text { pharmacokinetics. } \\
\text { Secondary objective: antitumor } \\
\text { activity. }\end{array}$ & $\begin{array}{l}\text { ORR: } 43 \% \\
\text { DOR: } 7.4 \text { months. } \\
\text { OS: } 12.3 \text { months. }\end{array}$ \\
\hline $\begin{array}{l}\text { EV-20I } \\
\text { NCT03219333 }{ }^{17}\end{array}$ & 2 & 125 & $\begin{array}{l}\text { Patients previously treated } \\
\text { with platinum-based } \\
\text { chemotherapy and CPI } \\
\text { therapy. } \\
90 \% \text { of enrolled patients } \\
\text { suffered from metastatic } \\
\text { visceral disease, while liver } \\
\text { metastases were present in } \\
40 \% \text { of patients. }\end{array}$ & $\begin{array}{l}\text { Primary end-point: ORR per } \\
\text { RECIST version I.I. } \\
\text { Key secondary end-points: DOR, } \\
\text { PFS, OS, safety, and tolerability. }\end{array}$ & $\begin{array}{l}\text { ORR: } 44 \% . \\
\text { Complete remissions: } 12 \% \text {. } \\
\text { DOR: } 7.6 \text { months. }\end{array}$ \\
\hline $\begin{array}{l}\text { EV-30I } \\
\text { NCT03474I } 07\end{array}$ & 3 & 608 & $\begin{array}{l}\text { Patients showed progression } \\
\text { of disease on either } \\
\text { platinum-based } \\
\text { chemotherapy or } \\
\text { immunotherapy. }\end{array}$ & $\begin{array}{l}\text { Primary outcome: OS. } \\
\text { Secondary outcomes: PFS, ORR, } \\
\text { DCR and DOR per RECIST VI.I, } \\
\text { assessment of safety and } \\
\text { tolerability, QoL and PRO } \\
\text { parameters. }\end{array}$ & $\begin{array}{l}\text { Significantly improves: } \\
\text { OS }(p=0.00 \mathrm{I}) \\
\text { PFS }(p<0.0000 \mathrm{I})\end{array}$ \\
\hline $\begin{array}{l}\text { EV-I03 } \\
\text { NCT03288545 }\end{array}$ & $1 / 2$ & 407 & $\begin{array}{l}\text { Assessment of } \mathrm{EV} \text { as } \\
\text { monotherapy or in } \\
\text { combination with anticancer } \\
\text { therapies such as } \\
\text { chemotherapy or CPI. }\end{array}$ & $\begin{array}{l}\text { Primary goal: safety, tolerability, } \\
\text { and efficacy of EV alone or in } \\
\text { combination with anticancer } \\
\text { therapies such as chemotherapy or } \\
\text { CPI. }\end{array}$ & $\begin{array}{l}\text { Initial results in } 45 \text { cisplatin- } \\
\text { ineligible patients: ORR per } \\
\text { RECIST I.I: } 62 \% \text { (I4\% with } \\
\text { complete and } 48 \% \text { with partial } \\
\text { response, respectively). } \\
\text { DCR: } 90 \% \text {. }\end{array}$ \\
\hline NCT03606I74 & 2 & 425 & $\begin{array}{l}\text { Participants previously } \\
\text { treated with CPI and } \\
\text { platinum-based } \\
\text { chemotherapy. }\end{array}$ & $\begin{array}{l}\text { Primary goal: safety, tolerability, } \\
\text { and efficacy of EV alone and in } \\
\text { combination with pembrolizumab } \\
\text { and/or chemotherapy. } \\
\text { The primary objective: ORR. }\end{array}$ & No results yet \\
\hline $\begin{array}{l}\text { EV-202 } \\
\text { NCT04225II } 7\end{array}$ & 2 & 240 & $\begin{array}{l}\text { Subjects with previously } \\
\text { treated locally advanced or } \\
\text { metastatic malignant solid } \\
\text { tumors. }\end{array}$ & $\begin{array}{l}\text { Primary purpose: ORR. } \\
\text { The study will also assess other } \\
\text { measures of antitumor activity } \\
\text { such as OS, safety and tolerability } \\
\text { of EV }\end{array}$ & No results yet \\
\hline EV-302 & 3 & 760 & $\begin{array}{l}\text { Patients with previously } \\
\text { untreated locally advanced } \\
\text { or mUC. }\end{array}$ & $\begin{array}{l}\text { Primary outcomes: PFS per } \\
\text { RECIST vI.I, OS. } \\
\text { Secondary outcomes: ORR, DOR, } \\
\text { DCR and TRAEs. }\end{array}$ & No results yet \\
\hline $\begin{array}{l}\text { EV-303 } \\
\text { NCT03924895 }\end{array}$ & 3 & 836 & $\begin{array}{l}\text { Cisplatin-ineligible } \\
\text { participants. }\end{array}$ & $\begin{array}{l}\text { Primary outcomes: PCRR (in all } \\
\text { participants as well as in those } \\
\text { whose tumors express PD-LI CPS } \\
\geq 10 \text { ), EFS (in all participants and in } \\
\text { those with EFS in tumors } \\
\text { expressing PD-LI CPS } \geq 10 \text { ). }\end{array}$ & No results yet \\
\hline
\end{tabular}

(Continued) 
Table I (Continued).

\begin{tabular}{|l|l|l|l|l|l|}
\hline Trial [Ref] & Phase & Patients & Inclusion Criteria & Endpoints & Outcomes \\
\hline NCT03869190 & $\mathrm{Ib} / 2$ & 385 & $\begin{array}{l}\text { Patients progressed during } \\
\text { or following a platinum- } \\
\text { containing regimen. }\end{array}$ & $\begin{array}{l}\text { Primary outcome: ORR. } \\
\text { Secondary outcomes: PFS, OS, OS } \\
\text { at specific time-points, DOR, DCR } \\
\text { and the percentage of participants } \\
\text { with AEs. }\end{array}$ & No results yet \\
\hline NCT0307099021 & $\mathrm{I}$ & 19 & $\begin{array}{l}\text { Japanese patients. } \\
\text { Post-chemotherapy setting } \\
\text { or ineligible for cisplatin. }\end{array}$ & $\begin{array}{l}\text { Assess the safety, tolerability and } \\
\text { pharmacokinetics, immunogenicity } \\
\text { as defined by the incidence of ADA } \\
\text { and anti-tumor activity. }\end{array}$ & $\begin{array}{l}\text { ORR: } 35.3 \% . \\
\text { DCR: } 76.5 \% .\end{array}$ \\
\hline
\end{tabular}

Abbreviations: ORR, objective response rate; DOR, duration of response; OS, overall survival; PD-LI, programmed-death ligand I; TRAEs, treatment-related adverse events; CPI, check-point inhibitor; RECIST, response evaluation criteria in solid tumors; PFS, progression-free survival; DCR, disease control rate; QoL, quality of life; PRO, patient-reported outcomes; mUC, metastatic urothelial cancer; PCRR, pathologic complete response rate; CPS, combined positive score; EFS, event-free survival; ADA, anti-drug antibody.

cycle) plus pembrolizumab, followed by standard of care surgery, followed by 6 cycles of postoperative EV plus pembrolizumab, followed by 8 cycles of pembrolizumab alone. This combination was compared to surgery alone and 3 preoperative cycles of pembrolizumab, followed by standard of care surgery, followed by 14 cycles of postoperative pembrolizumab. Each cycle is 21 days. The study started enrolling patients about a year ago and its primary outcomes are pathologic complete response rate (pCRR) in all participants as well as in those whose tumors express PD-L1 combined positive score (CPS) $\geq 10$ and event-free survival (EFS) in all participants and in those with EFS in tumors expressing PD-L1 CPS $\geq 10$.

\section{MORPHEUS MUC (NCT03869I90)}

$\mathrm{EV}$ is one of the drugs assessed in a phase $1 \mathrm{~b} / 2$, openlabel, multicenter, randomized, umbrella study in patients with locally advanced or mUC who have progressed during or following a platinum-containing regimen, with estimated enrollment of 385 patients. The study is designed with the flexibility to open new treatment arms as new treatments become available, close existing treatment arms that demonstrate minimal clinical activity or unacceptable toxicity, or modify the participant population (eg, with regard to prior anti-cancer treatment or biomarker status).

In this study that still recruits patients, participants receive atezolizumab and $\mathrm{EV}$ ( $\mathrm{EV}$ is administered at a dose of $1.25 \mathrm{mg} / \mathrm{kg}$ iv on days 1 and 8 of each 21-day cycle). In case of unacceptable toxicity or loss of clinical benefit, in terms of radiographic and biochemical data assessment, unfavorable local biopsy results or clinical status deterioration, the regimen administration is withdrawn. The primary outcome of the study is ORR, whereas secondary outcomes are PFS, OS, OS at specific time-points, DOR, disease control rate (DCR) and the percentage of participants with AEs. No results of the study are posted yet.

\section{NCT03070990}

It is an open-label, randomized, phase 1 study, designed to objectively assess the safety, tolerability and pharmacokinetics of EV when administered iv to Japanese patients with locally advanced or mUC in the post-chemotherapy setting or ineligible for cisplatin, as well as the immunogenicity as defined by the incidence of anti-drug antibody (ADA) and anti-tumor activity of EV. The estimated enrollment of the study is 19 participants. Patients were randomized $1: 1$ to receive $1.0 \mathrm{mg} / \mathrm{kg}$ (Arm A) or $1.25 \mathrm{mg} /$ $\mathrm{kg}$ (Arm B) EV on days 1, 8, and 15 of each 28-day cycle. According to initial results, EV was well tolerated across both doses, with dysgeusia and alopecia being the most common TRAEs, while the most common grade $\geq 3$ TRAEs were anemia and hypertension ( $\mathrm{n}=2$ each). ORR and DCR were $35.3 \%$ and $76.5 \%$, respectively. ${ }^{21}$

Tables 1 and 2 present a detailed summary of the aforementioned studies.

\section{Conclusions}

The therapeutic landscape for advanced and mUC continues to evolve. The recent regulatory approval of EV for the treatment of advanced urothelial cancer suggests a new, modern era, of precision therapeutics and confirms the evolving role of antibody-drug conjugates in solid tumors. EV shows a favorable profile in heavily pretreated 
Table 2 Adverse Events of Clinical and Ongoing Trials on EV

\begin{tabular}{|c|c|c|c|}
\hline Trial [Ref] & Phase & Patients & Adverse Events \\
\hline $\begin{array}{l}\text { EV-I0I } \\
\text { NCT0209199916 }\end{array}$ & I & 155 & 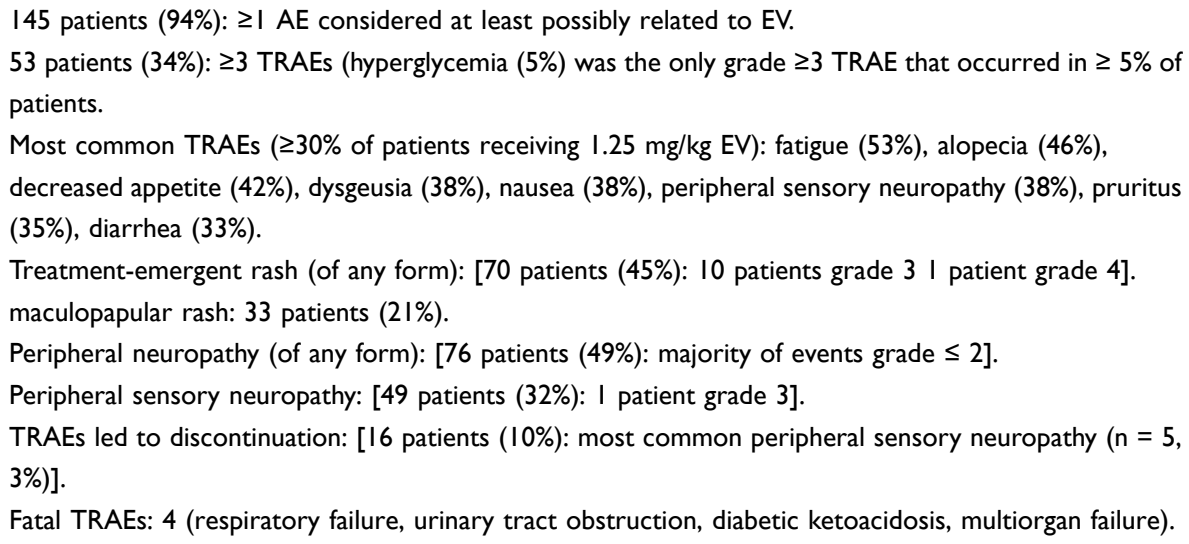 \\
\hline $\begin{array}{l}\text { EV-20I } \\
\text { NCT03219333 } 17\end{array}$ & 2 & 125 & $\begin{array}{l}\text { Most common TRAEs: fatigue ( } 50 \% \text { all grade, } 6 \% \text { grade } \geq 3 \text { ), alopecia ( } 49 \% \text { all grade), decreased appetite } \\
\text { ( } 44 \% \text { all grade, } 1 \% \text { grade } \geq 3 \text { ), dysgeusia }(40 \% \text { all grade, none grade } \geq 3 \text { ), peripheral sensory neuropathy } \\
\text { ( } 40 \% \text { all grade, } 2 \% \text { grade } \geq 3 \text { ). } \\
\text { Most common grade } 3 \text { or greater TRAEs: neutropenia }(8 \%) \text {, anemia }(7 \%) \text {, fatigue }(6 \%) \text {. } \\
\text { Most common serious TRAE: febrile neutropenia }(4 \%) \text {. } \\
\text { TRAEs led to dose reductions/discontinuation: } 32 \% / 12 \% \text { of patients [peripheral sensory neuropathy was } \\
\text { the most common TRAE led to dose reduction }(9 \%) \text { and discontinuation }(6 \%)] \text {. } \\
\text { TRAE peripheral neuropathy: [ } 50 \% \text { of patients, }(94 \%) \text { grade } 2 \text { or less]. } \\
\text { Peripheral sensory neuropathy: }(44 \%) \text {, motor neuropathy }(14 \%) \text {. } \\
\text { TRAE rash: }[48 \% \text { of patients, }(75 \%) \text { grade } \leq 2] \text {. } \\
\text { TRAE hyperglycemia: }[11 \% \text { of patients, }(50 \%) \text { grade } 2 \text { or less]. } \\
\text { No treatment-related deaths during the } 30 \text {-day safety reporting period. }\end{array}$ \\
\hline $\begin{array}{l}\text { EV-30I } \\
\text { NCT03474I07 }\end{array}$ & 3 & 608 & $\begin{array}{l}\text { Reported TRAEs were rash, hyperglycemia, decreased neutrophil count, fatigue, anemia and decreased } \\
\text { appetite (the most frequent Grade } 3 \text { or greater AEs occurring in more than } 5 \% \text { of patients). }\end{array}$ \\
\hline $\begin{array}{l}\text { EV- } 103 \\
\text { NCT03288545 }\end{array}$ & $1 / 2$ & 407 & $\begin{array}{l}\text { Most common TRAEs: fatigue }(66 \%, 14 \% \geq \text { Grade } 3) \text {, decreased appetite }(52 \%, 0 \% \geq \text { Grade } 3) \text {, alopecia } \\
(45 \%) \text {, diarrhea }(41 \%, 3 \% \geq \text { Grade } 3) \text {. } \\
\text { AEs of clinical interest: rash of any type }[45 \% \text { of patients }(14 \% \geq \text { Grade } 3)] \text {, peripheral neuropathy of any } \\
\text { type }[52 \%(3 \% \geq \text { Grade } 3)] \text {, immune-mediated events that required systemic steroid treatment }[17 \% \\
(10 \% \geq \text { Grade } 3)] \text {. } \\
\text { Treatment discontinuation: } 2 \text { patients }[(7 \%) \text { lipase increase, multi-organ failure]. }\end{array}$ \\
\hline NCT03606I74 & 2 & 425 & No results yet \\
\hline $\begin{array}{l}\text { EV-202 } \\
\text { NCT04225II7 }\end{array}$ & 2 & 240 & No results yet \\
\hline EV-302 & 3 & 760 & No results yet \\
\hline $\begin{array}{l}\text { EV-303 } \\
\text { NCT03924895 }\end{array}$ & 3 & 836 & No results yet \\
\hline NCT03869190 & $\mathrm{lb} / 2$ & 385 & No results yet \\
\hline
\end{tabular}


Table 2 (Continued).

\begin{tabular}{|c|c|c|c|}
\hline Trial [Ref] & Phase & Patients & Adverse Events \\
\hline NCT03070990 & I & 19 & 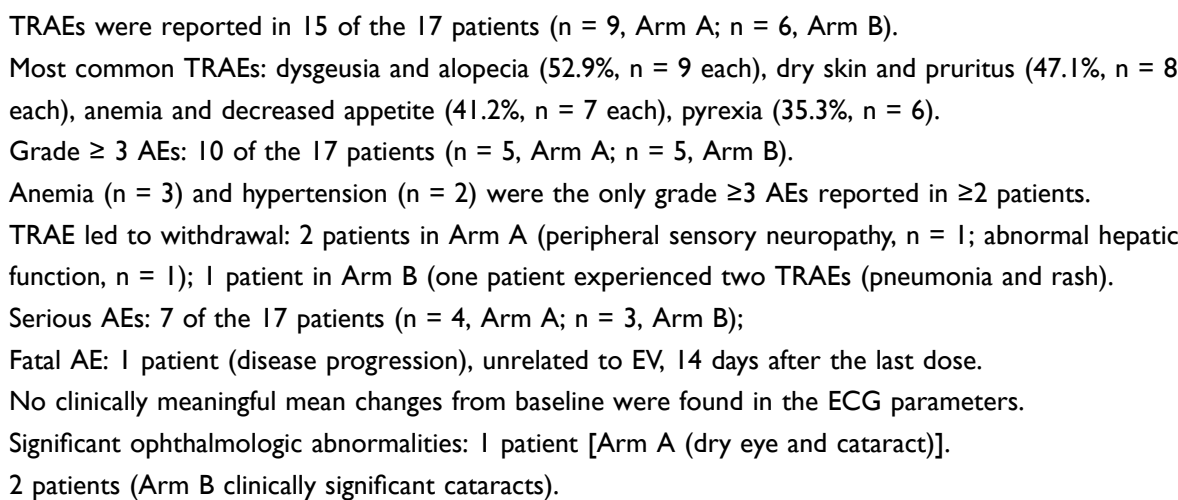 \\
\hline
\end{tabular}

Abbreviations: ORR, objective response rate; DOR, duration of response; OS, overall survival; PD-LI, programmed-death ligand I; TRAEs, treatment-related adverse events; CPI, check-point inhibitor; RECIST, response evaluation criteria in solid tumors; PFS, progression-free survival; DCR, disease control rate; QoL, quality of life; PRO, patient-reported outcomes; mUC, metastatic urothelial cancer; PCRR, pathologic complete response rate; CPS, combined positive score; EFS, event-free survival; ADA, anti-drug antibody.

patients with locally advanced or mUC. The available data is limited, however early survival reports demonstrate a significant antitumor effectiveness along with a rather acceptable safety profile in a difficult-to-treat population. Several clinical trials are ongoing in order to prove the possibly practice-changing role of $\mathrm{EV}$ advanced urothelial cancer.

\section{Expert Opinion}

In the recent years, the treatment paradigm of locally advanced and mUC has changed significantly, with the addition of CPIs and more recently with the FGFRtargeted therapies in our armamentarium. In 2019, EV achieved accelerated FDA approval in the post-platinum, post-CPI status of metastatic setting of disease as a result of its very encouraging and promising initial results in this special population of heavily pre-treated patients, even with unfavorable and poor prognostic factors, such as hepatic metastases. ${ }^{22}$

$\mathrm{EV}$ is currently assessed in several ongoing trials in other clinical scenario (EV compared with second-line chemotherapy) as well as in combination with other agents (pembrolizumab, immunotherapeutic agents or chemotherapy). At the moment, targeted therapies are the preferred second-line treatment options in the post-platinum setting or first-line treatment option for platinum-ineligible patients. ${ }^{23}$ According to NCCN guidelines, erdafitinib which is FDAapproved for the treatment of $\mathrm{mUC}$ patients with actionable FGFR3/FGFR2 alterations ${ }^{24}$ is recommended as second-line therapy after CPI or third-line therapy after platinum-based chemotherapy and $\mathrm{CPI}$, with $\mathrm{EV}$ recommendation as a preferred subsequent-line systemic therapy option. ${ }^{15,25}$ Both EV and erdafitinib are recommended treatment options in the post-platinum setting, however in everyday practice, the optimum sequencing protocol is still to be determined. FGFR alteration detection might be useful, in patients with progressive disease eligible for third-line therapy: for a patient with an FGFR2/3 alteration the choice could be based on possible TRAEs and their impact on patients' life.

Special consideration is needed regarding EV's toxicity. EV-related neurotoxicity and neuropathy affects approximately half of the patients treated with EV. Given the fact that several degrees of residual neuropathy are associated with cytotoxic agents given as first-line therapy, possible overlapping or deteriorating toxicities may render the administration of EV as second-line treatment difficult. ${ }^{26}$ Varying degrees of rash and severe eczematoid and lichenoid eruption are rather unusual, especially the cases with full-thickness epidermal necrosis or symmetrical drug-related intertriginous and flexural exanthema and are successfully treated with moisturizing agents and oral or topical corticosteroids. ${ }^{27,28}$

In this changing treatment landscape, there is great opportunity to develop and validate predictive biomarkers that can identify patients who are most likely to benefit from a particular therapy. ${ }^{29}$ Moreover, the optimal selection of best candidates for EV treatment remains a real challenge, while target expression and its biological factors such as selectivity, heterogeneity, internalization rate and functional role, are crucial in order to properly 
ascertain the safety, effectiveness and treatment potential of EV. ${ }^{9}$ Despite the optimistic perspective, there is still lack of data regarding the best timing of EV administration or sequencing and there is yet no standard of care in patients with advanced UC. ${ }^{30}$

\section{Disclosure}

The authors report no conflicts of interest in this work.

\section{References}

1. Ferlay J, Colombet M, Soerjomataram I, et al. Estimating the global cancer incidence and mortality in 2018: GLOBOCAN sources and methods. Int $J$ Cancer. 2019;144(8):1941-1953. doi:10.1002/ ijc. 31937

2. Teoh JY, Huang J, Ko WY, et al. Global trends of bladder cancer incidence and mortality, and their associations with tobacco use and gross domestic product per capita. Eur Urol. 2020;78:893-906. doi:10.1016/j.eururo.2020.09.006

3. Witjes JA, Bruins HM, Cathomas R, et al. EAU Guidelines on Muscle-Invasive and Metastatic Bladder Cancer. European Association of Urology (EAU); 2020

4. Cancer Stat Facts: Bladder Cancer. NIH NCI: surveillance, epidemiology and end results programme; 2019. Available from: https:// seer.cancer.gov/statfacts/html/urinb.html. Accessed January 19, 2021

5. Sternberg CN, Vogelzang NJ. Gemcitabine, paclitaxel, pemetrexed and other newer agents in urothelial and kidney cancers. Crit Rev Oncol Hematol. 2003;46(46 Suppl):S105-15. doi:10.1016/S10408428(03)00068-4

6. von der Maase H, Sengelov L, Roberts JT, et al. Long-term survival results of a randomized trial comparing gemcitabine plus cisplatin, with methotrexate, vinblastine, doxorubicin, plus cisplatin in patients with bladder cancer. J Clin Oncol. 2005;23:4602. doi:10.1200/ JCO.2005.07.757

7. Sternberg CN, de Mulder P, Schornagel JH, et al. Seven year update of an EORTC Phase III trial of high-dose intensity M-VAC chemotherapy and G-CSF versus classic M-VAC in advanced urothelial tract tumours. Eur $J$ Cancer. 2006;42(1):50-54. doi:10.1016/j. ejca.2005.08.032

8. Available from: https://www.fda.gov/news-events/pressannouncements/fda-approves-new-type-therapy-treat-advancedurothelial-cancer. Accessed February 02, 2021.

9. Boni V, Sharma MR, Patnaik A. The resurgence of antibody drug conjugates in cancer therapeutics: novel targets and payloads. Am Soc Clin Oncol Educ Book. 2020;40:1-17. doi:10.1200/EDBK_281107

10. Takai Y, Ikeda W, Ogita H, et al. The immunoglobulin-like cell adhesion molecule nectin and its associated protein afadin. Annu Rev Cell Dev Biol. 2008;24:309-342. doi:10.1146/annurev. cellbio.24.110707.175339

11. Jain RK, Skelton WP, Zhang J. Emerging treatment options for the treatment of metastatic urothelial cancer: therapeutic potential of enfortumab vedotin. Cancer Manag Res. 2020;20:8379-8386. doi:10.2147/CMAR.S224223

12. Challita-Eid PM, Satpayev D, Yang P, et al. Enfortumab vedotin antibody-drug conjugate targeting Nectin-4 is a highly potent therapeutic agent in multiple preclinical cancer models. Cancer Res. 2016;76:3003-3013. doi:10.1158/0008-5472.CAN-15-1313

13. Al Shaer D, Al Musaimi O, Albericio F, de la Torre BG. 2019 FDA TIDES (peptides and oligonucleotides) harvest. Pharmaceuticals (Basel). 2020;13(3):40. doi:10.3390/ph13030040
14. Padcev approval letter; 2019. Available from: https://www.access data.fda.gov/drugsatfda_docs/appletter/2019/7611370rig1s0001tr.pdf. Accessed October 19, 2020.

15. NCCN. Clinical practice guidelines in oncology (NCCN guidelines $^{\circledR}$ ). Bladder Cancer. 2020.

16. Rosenberg J, Sridhar SS, Zhang J, et al. EV-101: a phase i study of single-agent enfortumab vedotin in patients with nectin-4-positive solid tumors, including metastatic urothelial carcinoma. $J$ Clin Oncol. 2020;38(10):1041-1049. doi:10.1200/JCO.19.02044

17. Rosenberg JE, O’Donnell PH, Balar AV, et al. Pivotal trial of enfortumab vedotin in urothelial carcinoma after platinum and anti-programmed death/programmed death ligand 1 therapy. J Clin Oncol. 2019;37(29):2592-2600. doi:10.1200/JCO.19.01140

18. Maas M, Stuhler V, Walz S, Stenzl A, Bedke J. Enfortumab vedotin -next game- changer in urothelial cancer. Expert Opin Biol Ther. 2020;1-9. doi:10.1080/14712598.2021.1865910

19. Hoimes CJ, Rosenberg JE, Srinivas S, et al. 4844 - EV-103: initial results of enfortumab vedotin plus pembrolizumab for locally advanced or metastatic urothelial carcinoma. Ann Oncol. 2019;30 (suppl_5):v356-v402. doi:10.1093/annonc/mdz249

20. van der Heijden MS, Gupta S, Galsky MD, et al. 798TiP - study EV-302: a 3-arm, open-label, randomized phase III study of enfortumab vedotin plus pembrolizumab and/or chemotherapy, versus chemotherapy alone, in untreated locally advanced or metastatic urothelial cancer. Ann Oncol. 2020;31(suppl_4):S550-S550. doi:10.1016/j.annonc.2020.08.2069

21. Takahashi S, Uemura M, Kimura $T$, et al. A phase I study of enfortumab vedotin in Japanese patients with locally advanced or metastatic urothelial carcinoma. Invest New Drugs. 2020;38:1056-1066. doi:10.1007/s10637-019-00844-x

22. McGregor BA, Sonpavde G. Enfortumab Vedotin, a fully human monoclonal antibody against Nectin 4 conjugated to monomethyl auristatin E for metastatic urothelial Carcinoma. Expert Opin Investig Drugs. 2019;28(10):821-826. doi:10.1080/ 13543784.2019.1667332

23. Pistalmatzian N, Tzannis K, Pissanidou V, et al. Treatment of relapsed urothelial bladder cancer with vinflunine: real-world evidence by the Hellenic Genitourinary Cancer Group. Anticancer Drugs. 2016;27(1):48-53. doi:10.1097/CAD.0000000000000297

24. FDA. Resources for information on approved drugs. 2021. Available from: https://www.fda.gov/drugs/resources-information-approveddrugs/fda-grantsaccelerated-approval-erdafitinib-metastatic-urothelial -carcinoma. Accessed February 02, 2021.

25. Gopalakrishnan D, Koshkin VS, Ornstein MC, et al. Immune checkpoint inhibitors in urothelial cancer: recent updates and future outlook. Ther Clin Risk Manag. 2018;14:1019-1040. doi:10.2147/ TCRM.S158753

26. Grivas P, Drakaki A, Friedlander TW, et al. Conceptual framework for therapeutic development beyond anti-PD-1/PD-L1 in urothelial cancer. Am Soc Clin Oncol Educ Book. 2019;39:284-300. doi:10.1200/EDBK 237449

27. Sasaki R, Fujimura T, Lyu C, et al. Severe eczematoid and lichenoid eruption with full-thickness epidermal necrosis developing from metastatic urothelial cancer treated with enfortumab vedotin. J Dermatol. 2020;47:1436-1438. doi:10.1111/1346-8138.15577

28. Keerty D, Graham L, Haynes E, et al. Flexural exanthema from enfortumab vedotin. Cureus. 2020;12(5):e8102.

29. Nadal R, Bellmunt J. Management of metastatic bladder cancer. Cancer Treat Rev. 2019;76:10-21. doi:10.1016/j.ctrv.2019.04.002

30. Halford Z, Anderson MK, Clark MD. Enfortumab vedotin-ejfv: a first-in-class anti-nectin-4 antibody-drug conjugate for the management of urothelial carcinoma. Ann Pharmacother. 2020;106002802096040. doi: $10.1177 / 1060028020960402$ 


\section{Publish your work in this journal}

Drug Design, Development and Therapy is an international, peerreviewed open-access journal that spans the spectrum of drug design and development through to clinical applications. Clinical outcomes, patient safety, and programs for the development and effective, safe, and sustained use of medicines are a feature of the journal, which has also been accepted for indexing on PubMed Central. The manuscript management system is completely online and includes a very quick and fair peer-review system, which is all easy to use. Visit http://www. dovepress.com/testimonials.php to read real quotes from published authors. 